\title{
TIME AND PERIODICITY FROM PTOLEMY TO SCHRÖDINGER: PARADIGM SHIFTS vs CONTINUITY IN HISTORY OF MATHEMATICS
}

\author{
Yuri I. Manin
}

To Bob Penner, cordially

\begin{abstract}
I briefly consider the Kuhnian notion of "paradigm shifts" applied to the history of mathematics and argue that the succession and intergenerational continuity of mathematical thought was undeservedly neglected in the historical studies. To this end, I focus on the history of mathematical theory of time and periodicity, from Ptolemy's epicycles to Schrödinger's quantum amplitudes interference and contemporary cosmological models.
\end{abstract}

Keywords: Epicycles, Fourier series, quantum amplitudes, logarithm tables, Euler's number $e$.

AMS 2010 Mathematics Subject Classification: 01 A99

\section{Contents}

Introduction

1. Brief summary and plan of exposition

2. Mathematics and physics of periodicity

a. Antiquity: Euclidean geometry, Ptolemy's epicycles, Antikythera Digression 1: number $\pi$

b. Fourier sums and Fourier integrals: epicycles' calculus

Digression 2: number $e$ and "computational consciousness"

c. Quantum amplitudes and their interference

References 


\section{Introduction}

In his influential treatise [Ku70], Thomas Kuhn developed an approach to the history of natural science(s) based upon the assumption that this history can be naturally subdivided into periods. According to Kuhn, the transitions from one period to the next one (called "revolutions") are characterised by a radical change of the basic assumptions, experimental and observational practices, and acceptable types of argumentation. Any such set of assumptions is shared by the learned community during each development phase of "normal science", and its change is called a "paradigm shift".

Kuhn himself was reluctant about extending this view to the history, philosophy, religion, and much of the social science(s). He believed that they are formed rather by a "tradition of claims, counterclaims, and debates over fundamentals."

The motivation of this brief essay was a desire to discuss the applicability of the Kuhnian view on history of mathematics. I argue that the succession and intergenerational continuity of mathematical thought was undeservedly neglected in the sciencessical studies. To this end, I focus on the history of the the mathematical theory of time and periodicity, from Ptolemy's epicycles to Schrödinger's quantum amplitudes interference and Feynman integrals.

According to the concise description in [Da09], my essay lands somewhere in the uncharted territory between History of Science and Science Studies. Kuhn's book originated Science Studies "as a self-conscious field of inquiry" ([Da09], p. 801.) Hence this article belongs to it. But it focuses on the intrinsic continuity and the peculiarities of forms of historical legacy in understanding space, time, and periodicity that, for many historians, might be completely outside their fields of vision.

If one rejects, as I do here, the assumption about (this particular flow of) history as a sequence of revolutions, then the idea of paradigm shifts cannot claim anymore its leading role.

I accept here the more general viewpoint that Mathematics has a position mediating, or bridging, daily life, common sense, philosophy, and physics. Those fragments of mathematical knowledge that can become subjected to "reality tests" part are more sensitive to respective "revolutions" or "paradigm shifts", whereas those parts that are closer to "pure mathematics" show rather a kind of continuous development as is argued in this paper. Cf. also [Pa90], [Si12], [W10]. 
Also, some light on my position can be thrown by comparison of the history of developing knowledge on the scale of civilisations with the history of development of cognition in the individual brain of a growing human being (cf. [MM17].)

Acknowledgements. A project of this paper was conceived during a brief stimulating conversation of Yu.M. with Lorraine Daston about the role of Kuhn's "paradigm shifts" doctrine in the history of applied (or rather, "applicable") mathematics in a broad sense of this word.

Andreea S. Calude provided informative data about the prehistory of the cognitive behaviour of early Homo Sapiens.

When this paper was already written, Matilde Marcolli drew my attention to the article [Ga01] which contains a very careful and sensible survey of a considerable part of the same historical background (but excluding quantum mechanics, cosmology and my remarks on "computational consciousness").

I am very grateful to them for inspiring communication.

oritur sol et occidit et ad locum suum revertitur lustrans universa in circuitu pergit spiritus et in circulos suos revertitur

VULGATA CLEMENTINA, Ecclesiastes 1:5-1:6

\section{Brief summary and plan of exposition}

I will start with a few words about notions and formulas summarising some basic mathematical tools used in the contemporary discussions of time and periodicity.

Fundamental is the fact that these tools are subdivided into two complementary parts: geometric ones involving space/time intuition (as in Euclid's Elements) and algebraic/calculus ones involving formulae and computations and generally having linguistic character. Arguably (cf. [Ma15] and references therein) this is one reflection of the general dynamic patterns of interactions between right and left brain.

Start with an Euclidean plane $\mathbf{P}$ endowed with Euclidean metric. Then a choice of a point 0 , of a line $L$ passing through it, and of its orientation, determines an identification of the set of all points of this line with the set of real numbers $\mathbf{R}$ : this is its "coordinatisation". Call it the $x$-line and denote now $L_{x}$.

Choose now another line passing through the same point, oriented, and orthogonal to the $x$-line; call it $y$-line $L_{y}$. 
Now we can construct a "coordinatisation" of the whole plane $\mathbf{P}$ i.e., the identification of the set of points of $\mathbf{P}$ with the set of ordered pairs of real numbers $\mathbf{R}^{2}$.

At this point, we can start describing various figures, actors of plane Euclidean geometry, by equations and inequalities between various algebraic expressions involving $x$ and $y$. So, for example, a circle of radius $r$ whose center is a point $\left(x_{0}, y_{0}\right)$, is the set of all points $(x, y)$ whose coordinates satisfy the equation $\left(x-x_{0}\right)^{2}+(y-$ $\left.y_{0}\right)^{2}=r^{2}$.

We may call it Cartesian picture of geometry.

Similarly, using 3 coordinates one gets an algebraic picture of Euclidean geometry of space; passing to 4 coordinates, with time axis added to three space axes, we get the scene for Newtonian mechanics. But some fragments of this scene were already recognisable in the world pictures going back to the times of Archimedes and Ptolemy, as the celebrated Antikythera mechanism modelling the movement of heavens and relating them to the chronological dating of historical events. ([J17]).

Arguably, one important contribution of history of "periodicity" to mathematics was the crystallisation of the notions of "definition", initially emerging as secondary to the notions of "axiom" and "theorem" as in Euclid's "Elements".

The less obvious one was a "reification" of the idea of symmetry: statements and proofs of most theorems of Euclidean geometry are not dependent on the choice of origin of coordinates and therefore invariant with respect to parallel shifts of the whole space, and also with respect to rotations, conserving angles. Thanks to this, one can introduce "Cartesian" coordinates also on the space of all Euclidean symmetries of an Euclidean plane/space.

Finally, I must mention that, using the terminology of one of the schools of Science Studies, when I briefly quote and/or interpret mathematical intuition and historical data, I appeal mostly to "ethnomathematics in the European context" leaving aside many interesting achievements and inputs that came from Eastern, Chinese and other regions of the global world. For a much more complete and balanced treatment, see e. g. [Wo16]. 


\section{Mathematics and physics of periodicity}

\section{a. Antiquity: Euclidean geometry, Ptolemy's epicycles, Antikythera Mechanism.}

This book I bought in Venice for one ducat in the year 1507

Albrecht Dürer inscription in Euclid's book from his library

Before starting the central themes of our discussion, I must say explicitly that accumulation and intergenerational transmission of knowledge, became possible only at a certain stage of development of human language(s), and somewhat later, of written languages.

Moreover, as I argued in [Ma07], pp. 159-167 and 169-189, the most important new functions of emerging language consisted not in the transmission of concrete information about "here and now" ("in this grove a deer is grazing"), but rather in creation of "spaces of possibilities". Gods, heavens and netherworlds powerfully influenced human's collective behaviour, even if they could never be located here and now.

Since the concept of here and now itself later entered physical theories as coordinate origin, it would be interesting to trace its history as far back in time as possible. I am grateful to Andreea Calude who informed me that deep reconstruction (to about $15 \cdot 10^{3}$ years back from now) seemingly recovers old common Indoeuropean roots for "now" but not for "here", cf. [PagAtCaMe13]. Perhaps, a psychologically motivated substitution for "here" was furnished by very old ("ultraconserved") words for "I" and "you".

Passing now to the real origins of modern scientific knowledge about the Solar System and the Universe in the Greco-Roman and Hellenistic worlds, we see that its foundations were laid between $300 \mathrm{BCE}$ and $200 \mathrm{CE}$ and connected in particular with the names of Euclid, Archimedes, and Ptolemy. The history of "here and now", however, must alert us to the tracing also of the background history of the development of various new "languages of science", of translations and mutual interactions between these languages, and their intergenerational functioning.

Euclid of Alexandria conjecturally lived and worked at about $325 \mathrm{BCE}-265$ BCE in the south Mediterranean Greek colonial city. He created the richest and at that time logically perfect axiomatic description of two- and three-dimensional spaces with metric and their symmetry groups that were made explicit only many 
centuries later when the language of coordinates was created and one could speak about geometry using languages of algebra/calculus etc. Still, perception of Euclid's "Elements" as the foundational, almost sacral treatise survived till the 19th century: in particular new editions and translations of his Elements after spreading of printing were second only to the Bible.

See a very remarkable book [By1847] by Oliver Byrne, "surveyor of her Majesty's settlements in the Falkland Islands and author of numerous mathematical works", where he keeps texts of all his geometric chapters but rewrites all of Euclid's definitions (axioms), statements and proofs in pseudo-algebraic formulas in which traditional for us letters $a, d, c, \ldots, x, y, z$ serving as notations (for us) variables, constants, functions etc. are replaced by coloured pictures of angles, triangles et al.

Claudius Ptolemy conjecturally was born about 85 CE in Egypt and died about 165 CE in Alexandria, Egypt. His greatest achievement described in the "Almagest" is a dynamical model of the Solar System. This model is geocentric. This is justified by the fact that all our observation of planets and Sun are made from the Earth. It represents the visible movements of the planets and the sun as complex combination of uniform circular motions along epicycles, whose centres also move uniformly along their "secondary" epicycles, and finally various centres themselves are cleverly displaced from their expected ideal positions.

We do not know much about the computational devices that were used in antiquity in order to make Ptolemy's model and other models of observable periodicities such as lunar phases quantitatively comparable with observations. However, one remarkable archaeological discovery was made in 1900 when a group of sponger fishers from Greece during of bad weather anchored their boats near the island Antikythera and while they were diving discovered at a depth of 42 meters an ancient shipwreck. Besides bronze and marble statues, it contained a very corroded lump of bronze. All these remnants were transferred to the National Archaeological Museum in Athens, and after several decades of sophisticated studies and reconstructions, a general consensus arose summarised in [Sp08] as follows:

The Antikythera mechanism is an ancient astronomical calculator that contains a lunisolar calendar, predicts eclipses, and indicates the moon's position and phase. Its use of multiple dials and interlocking gears eerily foreshadows modern computing concepts from the fields of digital design, programming, and software engineering.

For a description of continuing disagreements about details of the reconstruction, see $[\mathrm{FrO2}]$ et al. 
Digression 1: the number $\pi$. In the history of geometric models of periodicity, the number $\pi$ plays a crucial role. Since Babylonian and Egyptian times, $\pi$ was considered ("defined") as the ratio of the length of a circle to its diameter that can be measured in the same way as other physical constants are measured. So in order to get an (approximate) value of $\pi$, one can first say, draw a circle using compasses, and then measure its length using a string. Independence of the result on the diameter is also an experimental fact which very naturally appears during land surveying. Finally, the approximate values are always rational numbers, or rather, names of some rational numbers, that can be transferred by means acceptable in the relevant culture: see a very expressive account by Ph. E. B. Jourdain [Jou1956] written at the beginning of the XXth century.

Arguably, the first modern approach to $\pi$ was found by Archimedes (about 287 $-212 \mathrm{BCE}$ ). This approach consisted in approximating $\pi$ from below by the values of perimeters of inscribed regular $n$-gons (diameter is for simplicity taken as unit of length). Manageable and fast converging formulas for consecutive approximations are obtained by passing from an $N$-gon to $2 N$-gon etc.

b. Fourier sums and Fourier integrals: epicycles' calculus. As we reminded in Sec. 1, after choosing orthogonal coordinates and scale identifying an Euclidean plane with $\mathbf{R}^{2}$, one can describe the circle of radius $r_{0}$ with centre $\left(x_{0}, y_{0}\right)$ as the set of points $(x, y)$ such that $\left(x-x_{0}\right)^{2}+\left(y-y_{0}\right)^{2}=r_{0}^{2}$. The variables change $x=r_{0}\left(x_{0}+\sin 2 \pi t\right), y=r_{0}\left(y_{0}+\cos 2 \pi t\right)$ describes then the movement of a point along this circle, with angular velocity one, if $t$ is interpreted as time flow. Replacing $t$ by $v_{0} t$ we can choose another velocity.

In turn, we can put in the formulae above $x_{0}=r_{1}\left(x_{1}+\sin 2 \pi v_{1} t\right), y_{0}=r_{1}\left(y_{1}+\right.$ $\left.\cos 2 \pi v_{1} t\right)$, in order to make the centre $\left(x_{0}, y_{0}\right)$ move along another circle with uniform angular velocity, etc. We get thus an analytic description of Ptolemy's picture, or rather its projection on a coordinate plane in our space, which can be complemented by projections on other planes.

In order to use it for computational purposes, we must input the observable values of $\left(x_{i}, y_{i}\right)$ and $v_{i}, i=0,1, \ldots$, for, say, planetary movements. The Antikythera mechanism served as a replacement of these formulae for which the language was not yet invented and developed. This language in its modern form and the analytic machinery were introduced only in the XVIII - XIX centuries: i.e. Fourier sums/series $\sum_{i}\left(a_{i} \sin i t+b_{i} \cos i t\right)$ and more sophisticated Fourier integrals were initiated by Jean-Baptiste Joseph Fourier (1768 - 1830). 
Joseph Fourier had a long and complicated social and political career starting with education in the Convent of St. Mark, and including service in the local Revolutionary Committee during the French Revolution, imprisonment during the Terror time, travels with Napoleon to Egypt, and office of the Prefect of the Department of Isère (where Joseph Fourier was born).

Returning briefly to Fourier's mathematics, I would like to stress also an analogy with Archimedes legacy, namely, observational astronomy and mathematics of his "Psammit" ("The Sand Reckoner"). Archimedes wanted to estimate the size of the observable universe giving an estimate of the number of grains of sand needed to fill it. Among other difficulties he had to overcome, was the absence of language (system of notation) for very large (in principle, as large as one wishes) integers. He solved it by introducing inductively powers of 10 , so that any next power might be equal to the biggest number, defined at the previous step.

Digression 2: the number $e$ and "computational consciousness". The famous Euler number $e=2,7182818284590 \ldots$ and his series

$$
e^{x}=1+\sum_{n=1}^{\infty} \frac{x^{n}}{n !}
$$

were only the last steps of a convoluted history, with decisive contributions due to John Napier (1550-1617, Scotland), Henry Briggs (1561-1630, England), Abraham de Moivre (1667-1754, France), among others, and finally Leonhard Euler (1707, Basel, Switzerland - 1783 St Petersburg, Russia).

As already with Archimedes, and later with the Masters of the Antikythera mechanism, one of the great motivations of the studies in this domain was the necessity to devise practical tools for computations with big numbers and/or numbers whose decimal notation included many digits before/after the decimal point/comma: this is what I call here "computational consciousness". This ancient urge morphed now into such ideas as "Artificial intellect" and general identification of the activity of neural nets with computations.

So, for example, Briggs logarithm tables allowing to efficiently replace (approximate) multiplications by additions consisted essentially in the tables of numbers $10^{7} \cdot\left(1-! 0^{-7}\right)^{N}, N=1,2,3, \ldots 10^{7}$. The future Euler's number $e$ was hidden here as a result of passing to the limit $e^{-1}=\lim \left(1-N^{-1}\right)^{N}, N \rightarrow \infty$, which Briggs never made explicit. However, the way Napier approached logarithms included approximate calculations of logarithms of the function sin, which made be considered 
as the premonition of the Euler formula $e^{i x}=\cos x+i \sin x$ that later played the key role in mathematical foundations of quantum mechanics.

c. Quantum amplitudes and their interference. As we have seen, the basic scientific meta-notions of "observations" and "mathematical models" explaining and predicting results of observations, go back to deep antiquity. The total body of scientific knowledge accumulated since then, was enriched during the XIX-th and $\mathrm{XX}$-th centuries also by recognition that "scientific laws", that is, the central parts of mathematical models explaining more or less directly the results of observations, are qualitatively different at various space/time scales: see a comprehensive survey ['t HoVa14], in particular, the expressive table on pp. 100-101.

A breakthrough in understanding physics at the very large scale Universe (cosmology) was related to Einstein's general relativity (or gravity) theory, whereas on the very small scale, the respective breakthrough came with quantum mechanics and later quantum field theory. Bridging these two ways of understanding Nature still remains one of the main challenges for modern science.

One can argue that an "observable" bridge between these two scales is the existence and cognitive activities of Homo Sapiens on our Earth (and possibly elsewhere), but the discussion of the current stage of "observations" and "explanations" in biology would have taken us too far away from the subject of this short essay; cf. [MM17]. Anyway, the key idea of scientific observation includes some understanding of how a subject of human scale can interact with objects of cosmic/micro scales.

Studying the small scale physics unavoidably involved the necessity of working out mathematical models of probabilistic behaviour of elementary particles that was observed and justified in multiple experiments. It was preceded by a remarkable cognitive passage: from the observable properties of chemical reactions to Mendeleev's intellectual construct of the Periodic Table to the images of atoms of the Chemical Elements as analogs of the Solar System with nucleus for Sun and electrons orbiting like planets. This cognitive passage might be compared with the evolution of astronomy from antiquity to Copernicus, Galileo and Newton.

When experimental methods were developed for working quantitatively with unstable (radioactive) atoms, small groups of electrons, etc., a new theoretical challenge emerged: observable data involved random, probabilistic behaviour, but the already well developed mathematical tools for describing randomness did not work correctly in the microworld! 
The emergent quantum mechanics postulated that "probabilities" of classical statistics, expressed by real numbers between 0 and 1, must be replaced in the microworld by probability amplitudes, whose values are complex numbers that after some normalisation become complex numbers lying in the complex plane on the circle of radius 1 and centre 0 . It must be then explained how to pass from the hidden quantum mechanical picture to the observable classical statistical picture. Many different paths along this thorny way were discovered in the 20's of the XX century, in particular, in classical works of Werner Heisenberg, Wolfgang Pauli, Erwin Schrödinger, et al. One way of looking at "quantization" of the simplest classical system, point-like body in space, is this. Any classical trajectory of this system is a curve in the space of pairs (position, momentum). Here position and momentum can be considered as Cartesian coordinate triples whose values, of course, pairwise commute. To the contrary, in the quantum mechanical space, the commutator between position and momentum is not zero. This can be envisioned as a replacement of possible classical trajectories of such a system by their wave functions which are not localised. Probabilistic data occur when one adds, say, one more point-like body and/or interaction with a macroscopic environment created by an experimenter.

Mathematical descriptions of all this are multiple and all represent a drastic break with "high school", or "layman", intuition. One remarkable example of pedagogical difficulties of quantum physics can be glimpsed in the famous Lectures on Quantum Mechanics by the great Richard Feynman.

In our context, the most essential is the fact that quantum interaction in the simplest cases of quantum mechanics is described via Fourier sums, series, and integrals in (finite dimensional) complex spaces endowed with Hermitean metrics, in place of real Euclidean space with real metric.

The quantum mechanical amplitudes are given by Fourier sums or series of the form $\sum_{n} a_{n} e^{i t}$ where $a_{n}$ are complex numbers, and $t$ is time, whereas probabilities in classical statistic descriptions are given by the similar sums with real $a_{n}$, and it replaced by inverse (also real) temperature $-1 / T$.

In this sense, quantum mechanics is a complexification of Ptolemy's epicycles.

In the currently acceptable picture, our evolving Universe can be dissected into "space sections" corresponding to the values of global cosmological time (e.g. in the so called Bianchi cosmological models) to each of which a specific temperature of background cosmic radiation can be ascribed. Going back in time, our Universe becomes hotter, so that at the moment of the Big Bang (time =0) its temperature 
becomes infinite. This provides a highly romantic interpretation of the correspondence $-1 / T \leftrightarrow$ it.

\section{References}

[By1847] O. Byrne. The first six books of the Elements of Euclid in which coloured diagrams and symbols are used instead of letters for the greater ease of learners, By Oliver Byrne surveyor of her Majesty's settlements in the Falkland Islands and author of numerous mathematical works. London William Pickering $184 \%$.

Facsimile publication by Taschen.

[Da09] L. Daston. Science Studies and the History of Science. Critical Inquiry, Vol. 35, No. 4, pp. 798-813. U. of Chicago Press Journals, Summer of 2009. https://doi.org/10.1086/599584

[Fr02] T. Freeth. The Antikythera mechanism. Mediterranean Archaeology and Archaeometry, vol. 2, no. 1, pp. 21-35.

[Ga01] G. Gallavotti. Quasi periodic motions from Hipparchus to Kolmogorov. Rendiconti Acc. dei Lincei, Matematica e Applicazioni., vol. 12, 2001, pp. 125-152.

[Gr17] St. Greenblatt. The rise and fall of Adam and Eve. W. W. Norton \& Company, 2017.

['t HoVa14] G. 't Hooft, St. Vandoren. Time in powers of ten. Natural Phenomena and Their Timescales. World Scientific, 2014.

[J17] A. Jones. A portable cosmos: revealing the Antikythera mechanism, scientific wonder of the ancient world. Oxford UP, 2017.

[Jou1956] Ph. E. B. Jourdain. The Nature of Mathematics. Reproduced in the anthology "The World of Mathematics" by James R. Newman, vol. 1, Simon and Schuster, NY 1956.

[Ku70] Th. Kuhn. The Structure of Scientific Revolutions (2nd, enlarged ed.). University of Chicago Press, 1970. ISBN 0-226-45804-0.

[Ma15] Yu. I. Manin. De Novo Artistic Activity, Origins of Logograms, and Mathematical Intuition. In: Art in the Life of Mathematicians, Ed. Anna Kepes Szemerédi, AMS, 2015, pp. 187-208.

[Ma07] Yu. Manin. Mathematics as Metaphor. Selected Essays, with Foreword by Freeman Dyson. 
[MM17] D. Yu. Manin, Yu. I. Manin. Cognitive networks: brains, internet, and civilizations.. In: Humanizing Mathematics and its Philosophy, ed. by Bh. Sriraman, Springer International Publishing AG, 2017, pp. 85-96. DOI 10.1007/978-3319-61231-7_9. arXiv:1709.03114

[PagAtCaMe13] M. Pagel, Q. D. Atkinson, A. S. Calude, A. Meade. Ultraconserved words point to deep language ancestry across Eurasia.

www.pnas.org/cgi/doi/10.1073/pnas.1218726110

[Pa90] D. Park. The How and the Why. An essay on the origins and development of physical theory. Princeton UP, New Jersey, 1990.

[Si12] K. Simonyi. A Cultural History of Physics. Translated by D. Kramer. CRC Press, Boca Raton, London, New York, 2012.

[Sp08] D. Spinellis. The Antikythera Mechanism: a Computer Science Perspective. Computer, May 2008, pp. 22-27.

[W10] G. Wolf. Orrery and Claw. London Review of Books, 18 Nov. 2010, pp. 34-35.

[Wo16] D. Wootton. The Invention of Science: A New Histrory of the Scientific Revolution. Penguin, 1976, 784 pp.

Max-Planck-Institute for Mathematics, Vivatsgasse 7, Bonn 53111, Germany.

manin@mpim-bonn.mpg.de 